Proceedings of the International Scientific Conference. Volume II, May $22^{\text {th }}-23^{\text {th }}, 2020.280-291$

\title{
FEATURES OF THE USE OF EMODIA IN THE STUDY OF HUMANITARIAN DISCIPLINES AT THE EXAMPLE OF HISTORY
}

\author{
Oleksandr Romanukha \\ Donetsk National University of Economy and Trade named after Mykhailo \\ Tugan-Baranovsky, Ukraine \\ Hannah Udovichenko \\ Donetsk National University of Economics and Trade named after Mykhailo \\ Tugan-Baranovsky, Ukraine \\ Viktoriia Zinchenko \\ Donetsk National University of Economics and Trade named after Mykhailo \\ Tugan-Baranovsky, Ukraine
}

\begin{abstract}
This article deals with the potential to use emoji in the studying process. The purpose of the article is a determination of the potential for use of emoji in the educational process as a partial substitute of the traditional text. There were used the following methods to solve the desired goal: the analysis of the scientific works regarding the matters under the inquiry, synthesis of ideas; generalization of the own pedagogical experience; method for description of the interactive forms of teaching during seminars and lessons in secondary school. The author of this article created the algorithm to use emoji in the history classes at school and university. According to the results of poll among pupils and students emoji is more popular in secondary school. During the classes emoji can be used in various ways: partial emoji translation of the text; emoji testing; quest; using emoji in literary Internet projects. Using emoji has methodical, psychological and technical advantages such as the decrease of the volume of the educational material; the increase of the emotional interest of pupils and students; the development of the logical and associative thinking; conducting classes by dint of smartphones and messengers; distance learning. Using emoji in the studying process engages not only innovation technologies, but also higher nervous system of person, the language of symbolic logic.
\end{abstract}

Keywords: educational process, emoji, informational technologies, pedagogy

\section{Introduction}

Society and economy reckon on highly competent people while competence requirements are changing; not only good basic skills (literacy, numeracy and basic digital skills) and civic competences; skills such as critical thinking, initiative taking and problem solving, creativity play an increasing role in coping with complexity and change in today's society. The development of 
informational technologies promotes the transformation of education: a diversity of study, simplification of a procedure for receiving of the necessary information, its processing. Competences are not static; they change across generations and throughout life. It is more important convince that all people of different ages have the opportunity to get the better of the required competences in initial education and training, higher education or different forms of nonformal and informal learning. The use in the educational process of the computer and internet generated: presentations, electronic textbooks, onlinetrainings. Extending to the educational process, technical progress, it produces more and more new approaches, influencing the foundations of our thinking. In the previous publication "Graphical interface as a didactic component of an electronic text-book" (Romanukha, 2018) it was a question about the importance of transformation of principles for composition of electronic text-books and the influence of the informational technologies on the change of people's thoughts, principles of data processing, especially among the youth. Continuing the development of the problem, it is proposed to pay attention to the matters of integration of languages of a human and a machine within the boundaries of the information space. As modern technologies bring together thinking, speech, the principles of information perception of a human with a machine, it increases the need to update the principles of work with text as the main source for saving and transferring of information.

The use of text in the educational process is dominant till the present time. The receiving of new information, communication through the electronic mails, messengers and other communicators are also based on the text. The super saturation of the text in people's life decreased its interest to reading and promoted the transfer to the simpler forms of communication - smiles, emoji. Their appearance was rapidly changing the world around, and the use reflected in many aspects was reflected in many aspects of human existence: personal life; literature; communication; art, cinema; marketing and so on. Today, emoji is one of the unofficial, but widespread electronic languages of communication, which are used in many countries of the world with different religions, languages, culture.

It is only the beginning of the use of emoji in education. Now the only country that has declared its intention to allow officially emoji in the educational process at the level of kindergartens is Australia. The teachers held that their use is quite argued and well-timed. Thanks to their brightness and unusualness, they are able to capture and attract attention of children with different motivations. The use of smiles allows to estimate and to transfer faster an information than with usual language or text. The smiles save time on encoding and decoding of information, as it is more recognizable. The advantage of using of smiles and 
emoji lies in the direction of inclusive education aimed at children with certain developmental disadvantages.

The purpose of the article is a determination of the potential for use of emoji in the educational process as a partial substitute of the traditional text.

There were used the following methods to solve the desired goal: the analysis of the scientific works regarding the matters under the inquiry, synthesis of ideas; generalization of the own pedagogical experience; method for description of the interactive forms of teaching during seminars and lessons in secondary school.

We have studied using of emoji in educational process of Donetsk National University of Economics and trade named after Mykhailo Tugan-Baranovsky in Kryvyi Rih city (60 persons, among them were students from specialty Philology, who study some themes from History in English and students from other specialties with sufficient level of knowing English) and Kryvyi Rih secondary school No.10 (50 persons). During the classes emoji can be used in various ways: partial emoji translation of the text; emoji testing; quest; using emoji in literary Internet projects. According to the results of poll among pupils and students emoji is more popular in secondary school.

\section{Historical Development of Emoji}

A study of this question is reflected in a number of publications. Emoji, as a means of nonverbal youth communication, was studied by O. Dubrovska. The author emphasizes that an informatization leads to the fact that the Emoticans, being used for thought-transfer, become as a full-fledged replacement of words and letters (Dubrovskaya, 2016). The scientists K. Osadcha and H. Chemerys emphasize the importance of use of symbols in analysis of instrumentarium for prototyping of a program interface (Osadcha \& Chemerys, 2018). A positive result for use namely of emoji in the educational process was emphasized by the teacher Marissa King (Marissa, 2018). She thinks, that gives a better understanding of those fact that a lot of in the verbal speech also depends on context. H. Ukhanaieva speaks about emoji as a new language of the virtual space, which is actively spreading around the world (Ukhanaieva, 2018). O. Kitova agrees with such conclusions (Kitova, 2016). She argues that emoji and emoticans perform an orienting function in communication and they dominate in the certain communities.

The emoji language appeared in Japan at the end of the XX century. Firstly, it was a language of ideograms and smiles, which were used on the web-pages and in the electronic massages. However, soon it captivated all the world. As the result, it was firstly officially placed an announcement for a vacancy of translator of smiles (emoji) in 2016 in London. A pictogram communication is 
known to the humanity from the ancient times: cave drawings, wedge writing, hieroglyphic symbols of the ancient Egypt, writing of Maya tribes, etc. Namely the use of pictograms was effective for the longtime saving of information, its coding in the large volumes. However, it wasn't possible for the humanity to refuse from the signs, as from the way of communication, even in the XXI century. The space exploration, an appearance of radio-communication, of internet, of artificial intelligence not only saved, but also they gave an impulse to the further development: road signs, aviation emergency gesture signaling and other (Fig. 1)

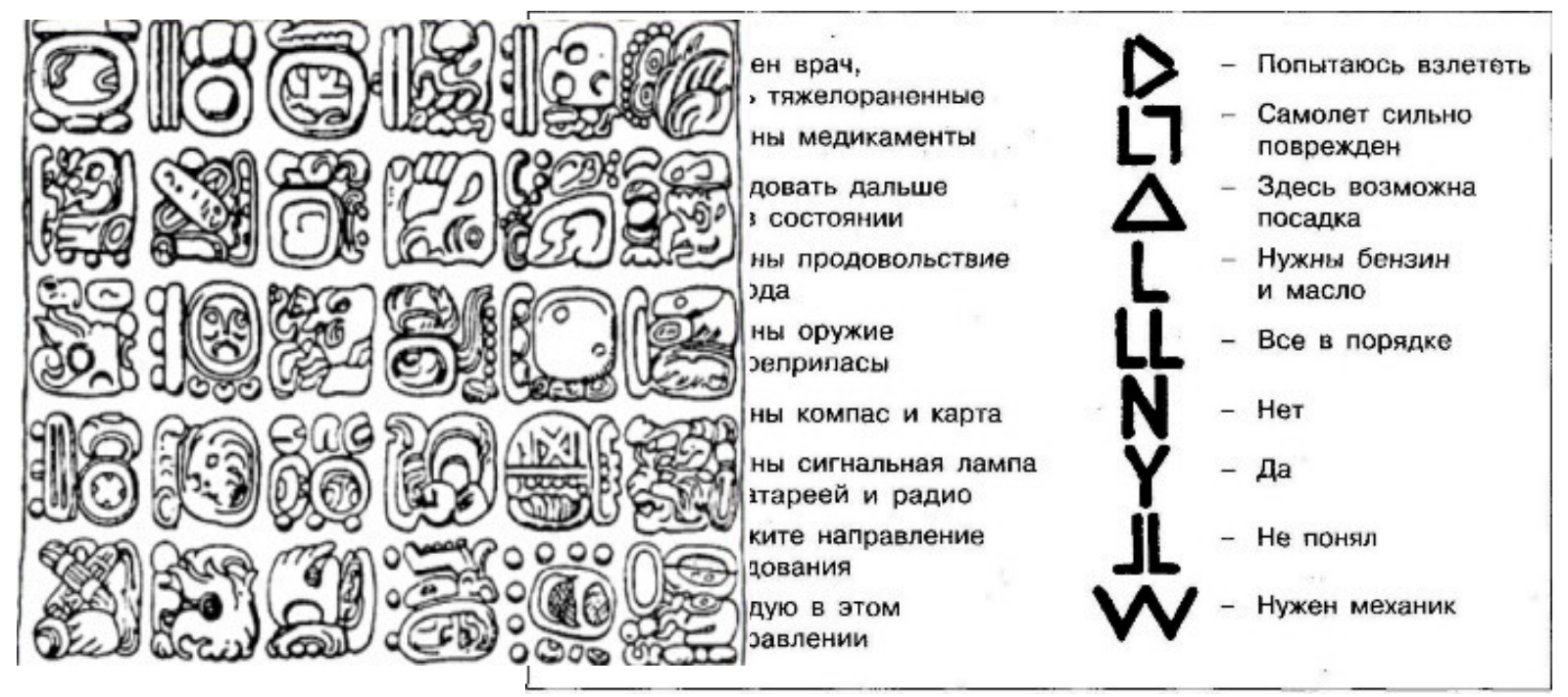

Figure 1 Writing of Maya; Aviation emergency gestures signaling (Nosyrev, 2018)

\section{Emoji Application Today}

Therefore, we can surely say about the pictogram, the symbol of emoji - as a code system for processing of information, which is used for the change of name of an object with a sign to provide more comfortable and effective processing of information, its transmission, saving. Considering the fact that it is emphasized in the science three forms of coding: sound, numeric, graphic, we will talk about the last one. The first coding systems - language, arithmetic played a great role in the human progress. However, globalization requires the development of a common language, a system of communication. With time this place take emoji. They are understandable for representatives of different races, cultures, are actively used in marketing, culture, communications. A wide group of users of such language are children of school age, young people, people who are active users of the Internet space, computers, smartphones. In the context of information coding these principles are actively used in schools. Trying firstly to teach a child to read and to write, the teacher inculcates to this child the adjusted 
code systems for information processing. It is occurred a building of other on their basis and etc. A remarkable example for code, symbol use is evaluation in the senior school with the grades, and in the junior school with signs (smile, sun etc). The spread of technologies, which lead to the increase of information flow, density of communication only promote the development of emoji language. Marshal Makliunen thinks that, "these processes return humanity to a tribal state - tribalization. People in the live chats use an informal style of language in the verbal conversation, as primitive people transmit news - however, not from mouth to mouth, but from chat to chat. The society of modern age, which practices an oral speech in writing with the help of technology, is called postwriting” (Ukhanaieva, 2018).

However, if such changes occurred earlier during millenniums, centuries, then today these phases are measured with the decades, and sometimes with years. A language evolution occurs at a quick rate. So the "Yandex: translator" taught to translate texts into emoji and conversely ("Yandex. Translator» learned Emoji, 2018). The users of iPhone and iPad received the possibility with a new function - Spotlight in iOS to make a search in the Internet by the smiles (Spotlight in iOS supports emoji search, 2018). As a result, the list of the used emoji rises fast. Therewith, the companies themselves are launching mobile apps to search for emoji around you. The company Google has released the program Emoji Scavenger Hunt, which works thanks to the neuronet and it helps the user to search emoji around themselves. Emojization captivated the world (Google has released a game for searching emoji around itself, 2018).

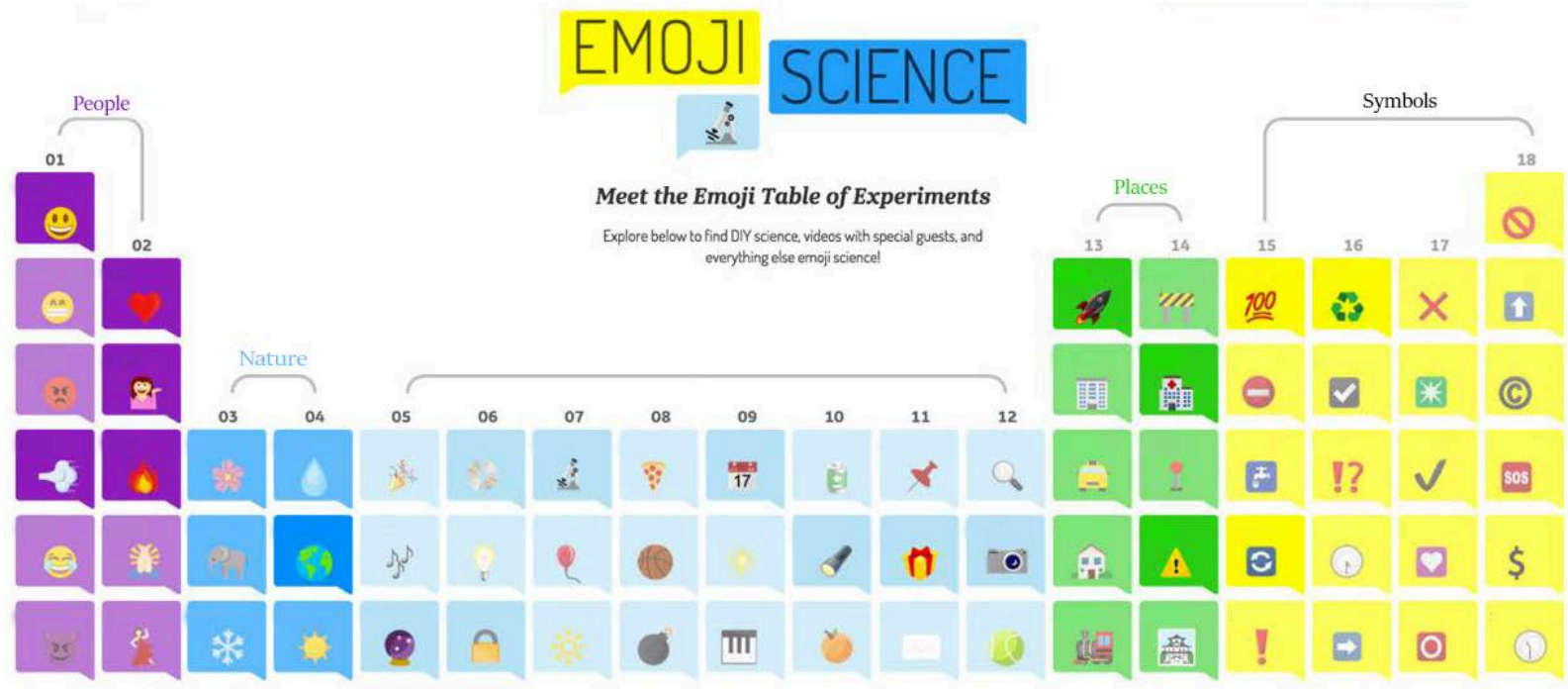

Figure 2 Table of experiments General Electric 
The directions and spheres for the practical use of emoji are enough wide. They are already widespread in the form of educational content. The company General Electric on emojiscience.com placed a table of experiments in the view of periodic table, where all information was encoded in emoji. It was necessary for the user to push on the certain smile to watch an experiment (Kane, 2018). If you wished to see a rocket firing, it was necessary to push on the image of a rocket (Fig. 2).

A wide spreading of emoji was in marketing. All modern advertisement on the basis of messengers and Internet is built only on emoji (See figure 3).

Dear passengers! ON\$ This is an announcement that Flight 2019 has started boarding ! Your baggage $\hat{\Omega}$, has to have only the pleasant souvenirs from $20180098 \%$. Attention

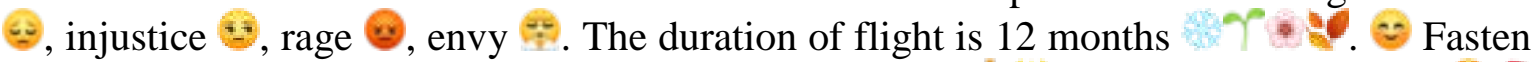
your seat belt, the next stop is a "Year of happiness» $\mathcal{L}_{2}$. Have a nice flight! Happy upcoming New Year! $4508 \%$

Figure 3 Example of emoji-advertisement of travel company, which is sent to its clients through Viber

The borrowing of the principles and methods for use of emoji in various fields opens the wide opportunities for their implementation in the educational process. The poll, published on the website Dictionary.com, found that, 37 percent of teachers use emoji, gifs and memes on their lessons. In such a way teachers try to make the educational material more interesting and to attract the attention of teenagers. Most often they are used on lessons in secondary school 44 percent of teachers use them there. In college and university, they are used by 33 percent of teachers, and only by 29 percent in elementary school (Kondratieva, 2018). The directions for use: motivation; designation of subject; distribution of roles during team working; emotional accompaniment and etc.

The consideration of current trends and forms for use of emoji promoted the development of the emoji-oriented complex of knowledge. The author foreseen a wide use of emoji in different forms of works: from the text to the execution of exercises for consolidation of learned material, examination of acquired knowledge:

- $\quad$ Reading of the text with elements of emoji;

- $\quad$ historical emoji mathematics. A group of emoji + other group of emoji

$=$ event which should be indicated;

- $\quad$ to put in a missed word - emoji; 
- $\quad$ open task to recode text in emoji. Rewriting of letters from the past;

- $\quad$ quizes - varieties of guessing games.

The technical means for realization of assigned tasks became messengers in the mobile devices. Let's look at certain types of work in more details. "The reading of text with elements of emoji”, the advantage of the offered method consists in: reduction of material volume in saving of the semantic load; the interest of work with text; originality of material presentation; easiness of remembering and associations setup; creative thinking; awakening of motivation to the associative thinking; the disappearance of the problem of student's distraction on the phone. The experiment showed that the correlation of emoji with text has to be not more than 30/70\% depending on the topic and complexity of the text.

The optimal index is $-20 / 80 \%$. A huge oversaturation with emoji leads to complication to understand the sense and to the loss of interest. An increase of the percentage of emoji is possible upon condition of their systematic addition, taking into account the individual abilities and possibilities of the students. Herewith, the work with text has to be based on the principle of clear definition of the meaning emoji and on the further permanence of their role. The emoji have not to be applied to names, surnames, terms (Table 1).

\section{Table 1 A comparative table of texts with and without use of emoji}

\begin{tabular}{|c|c|}
\hline MERE TEXT & TEXT WITH USE OF EMOJI \\
\hline $\begin{array}{l}\text { In May 1803, the First Consul directed } \\
\text { the French Army to Weser, to capture the } \\
\text { belonging to the King of England Hannover; } \\
\text { in June the electorate was already under the } \\
\text { reign of France as a result of cowardice of } \\
\text { the local authority, which hurried to } \\
\text { conclude with the First Consul an } \\
\text { agreement, according to which the French } \\
\text { Army could occupy all country to the } \\
\text { territory of Elba, and the Army of Hannover } \\
\text { had to be dismissed. On May 18, } 1804 \\
\text { Napoleon was acclaimed as emperor of } \\
\text { Frenchmen - Napoleon the I }{ }^{\text {st }} \text {. He began to } \\
\text { regroup the forces on the north of France (in } \\
\text { the Boulogne camp) to organize the forcing } \\
\text { of the English Channel and to land the } \\
\text { army's expedition in the Great Britain. The } \\
\text { worried with it Englishmen began an active } \\
\text { diplomatic activity for creation of a new } \\
\text { coalition against Napoleon I. The Russian } \\
\text { Empire concluded with Great Britain the St. }\end{array}$ & 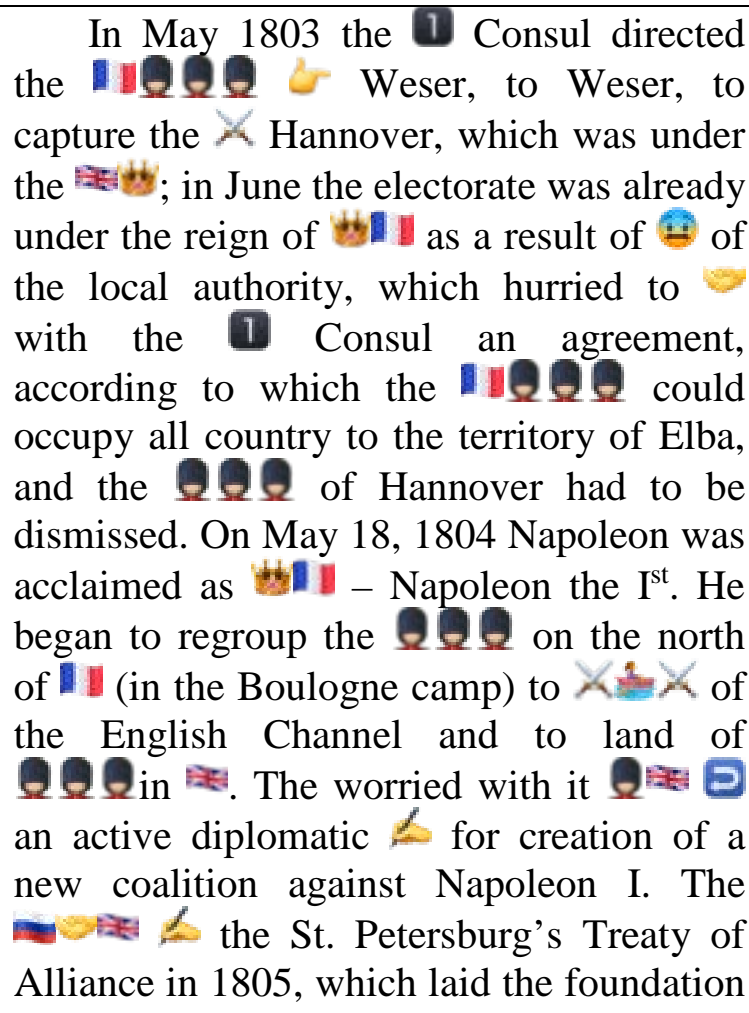 \\
\hline
\end{tabular}


Petersburg's Treaty of Alliance in 1805, which laid the foundation of the $3^{\text {rd }}$ antiFrench coalition. In the Trafalgar fight in 1805, the united Franco-Spanish naval forces had the worse from the British squadron under the command of admiral G. Nelson. It broke the plans of Frenchmen by invasion in the Great Britain. of the 3 A II coalition. In the Trafalgar in 1805, the united 1 from the -18 G. Nelson. It the plans of by $X$ in the

We use this type of work when conducting seminars on the subject "History of Ukrainian Statehood and Culture" and in the course of teaching "World History" in a comprehensive school. This text is offered as a template for creating your own text with elements of a discipline topic. $70 \%$ of students are interested in this type of work. Creating your own text with elements of emoji is closely related to the self-realization and level of media literacy, and between the self-actualization of the individual and his level of media competence, and is one of the important trends of modern humanities. The results of our own experience are described in more detail below.

The following example is a historical emoji mathematics. Its use is appropriate after insight into the theme's material. She will help for the better remembering of events, personalias, facts. Such format for material consolidation can be presented in the form of popular game - quiz. It is given to a participant a set of emoji, and he/she has to guess an event, person or name. As a help, you can give an informational resource zaxid.net which proposes to print the meaning of emoji and to indicate about which famous Ukrainian it is talked about (Table 2) (All on emoji. Festive test of Ukrainians in emoticons and symbols, 2018).

Table 2 A comparative table of task for consolidation of the studied material: emoji-quiz; emoji mathematics; to put in a missing word

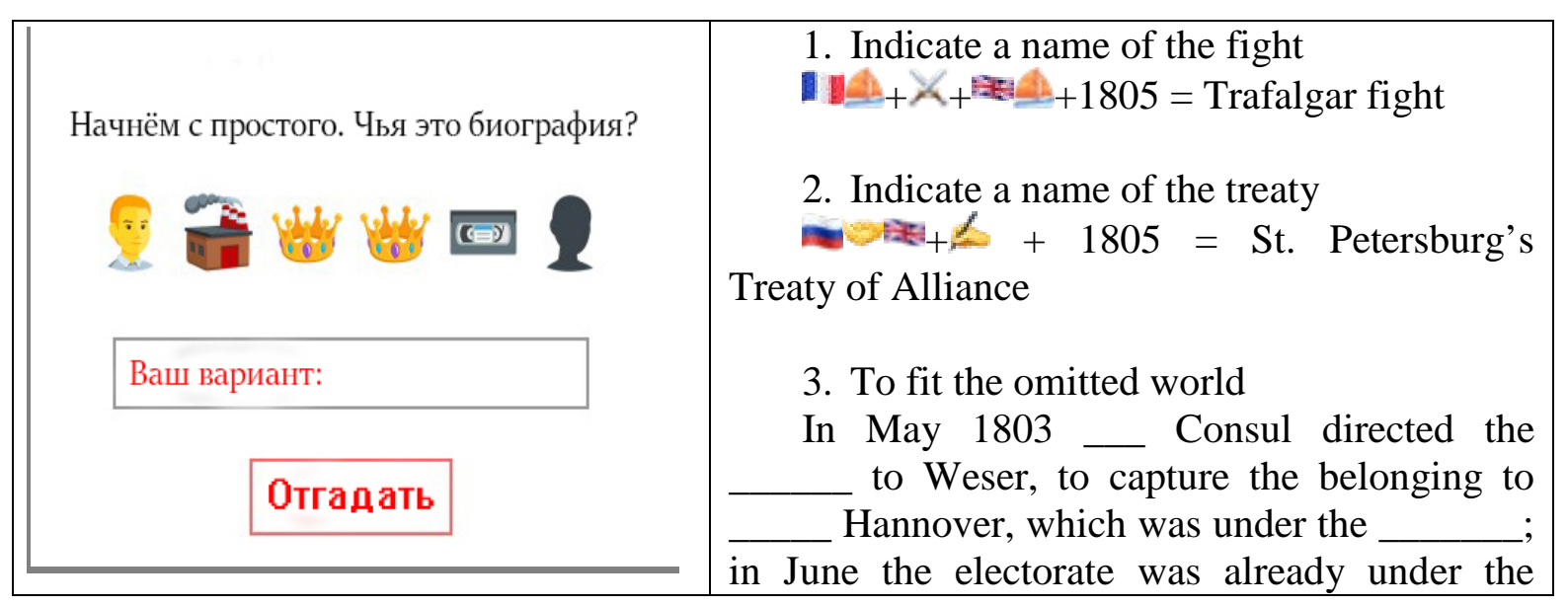




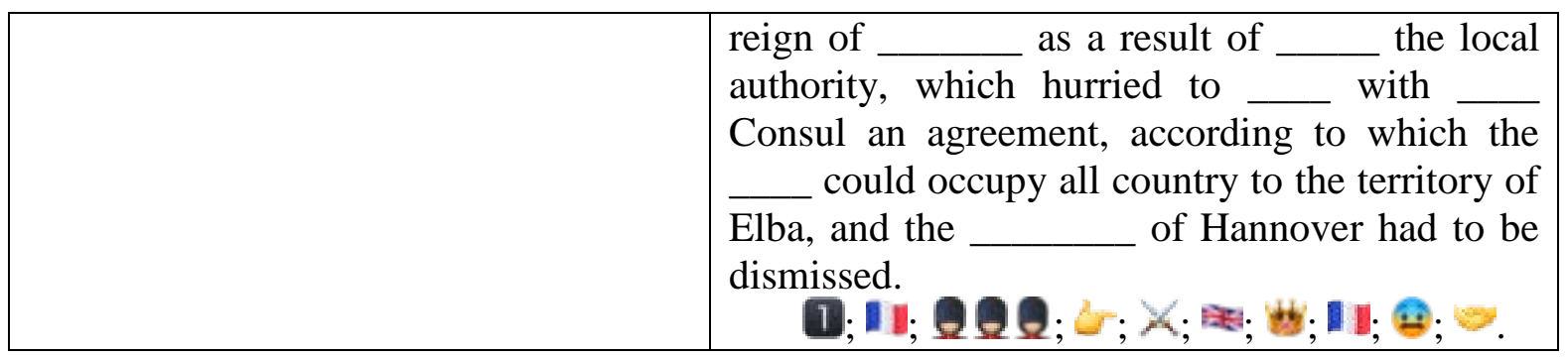

The rewriting of the letters of past. To ask students to rewrite the letter of the past with words and symbols of modernity, to imagine what the person of those times felt and wanted to say. It is interesting. The children will think about the hero's feelings, as in mind they will "bring him/her closer”, place him/her in their space, and see the real person.

The similar principles have been already used in literature not the first year and. In such a way an informational resource platfor.ma offers to try to read on the emoji language and to the contrary a creative work of T.H. Shevchenko. Herewith the website of the portal was built in its design with the use of significant number of emoji. A long time the similar projects have been walking around the world. With the purpose to popularize the creative work of O. Pushkin and B. Pasternak in the world, it was even developed a special project and program "Emoji-Pasternak". According to the showed results, these developments make the study of literature more interesting, especially in study of poems. The realization of analogies with symbols helps to remember words in better way.

The use of modern informational systems gives a possibility to execute and estimate such kinds of work without reference to the place or some special program. In this case as technical means can serve simple smartphones, and as the dialog platforms can serve the social nets, messengers (Viber, WhatsApp, Facebook Messenger), Skype, etc.

The teachers from different countries of the world agree with the principles and forms for use of emoji in the educational process. Marissa King, a teacher from Oklahoma notes: "Ask students to analyze examples in which the same smiles are used in different meanings." For this, they look at the messages in a screenshot from Instagram or YouTube, which includes pictures and texts. In such a way, the pupils understand that in verbal speech a lot also depends on the context. In addition to it, it can be proposed to develop the usage guidelines for usage of emoji for beginners: the pupils will list different meanings, offer examples and explain how to determine what exactly it is intended in different messages”. Such tasks, according to the Maris's opinion, help to find a connection of school education with the everyday practical trainings of pupils and, in such a way, to make the study more significant (Marissa, 2018). 
The use of emoji expands the psychological, methodological, technical teacher's tools. In the context of informatization, they give a possibility not to separate the study from the reality, the peculiarities of youth communication. A sign, a symbol is always is always perceived more positively and better than just the text. A symbol attracts attention, makes the text more interesting, activates thinking, imagination. In the study of history it is very important, as the teacher's task is not only to transfer the knowledge, but to form a critical thinking, an image. At the same time, a symbol helps to a person to perceive a sensation, which are transferred without any contact, to include own experience and emotions in seconds. To understand an emotional orientation of the text, it is enough to look at a symbol, but not to read several pages. The scientists explain the success of emoji with their closeness to a unified ancient language. According to Zimmer Frensis, there is an old utopian ideal - the universal illustrative speech. It is often compared with a language of pre-Babylonian times. The scientist himself was actively developing similar characters in the 50s of the XX century. He explained the preconditions of such actions as natural return to the basics, primary sources.

Herewith, it should be expressly understood that the oversaturation of text with emoji, the lack of clear regulations for their use will lead to the loss of the text's essence, its incomprehensibility, negative attitude to the subject, and learning in general. The principle of replacement of words with emoji works only with short sentences, which have a lack of their own names. As showed the results of research, the percent of replacement of words with emoji, in average, has to be no more than 30\%. An expansion in a number of emoji leads proportionally to the strengthening of complexity in text understanding. Therefore, the main message of emoji is not so much to replace the words, texts, as to make them more interesting and simple for study. A reason for a clear percentage of emoji in the text lies in the fundamental difference of ideograms and pictograms. The ideogram is read in a natural verbal way, and the elements of ideographic writing are essentially words, speech signs. A pictogram is an image, which can be interpreted in many ways. It can be interpreted as a verb, as a noun, and as an adverb. More simply this is an idea, which is able to accept various verbal expressions. For example, if you draw a bus, then it can mean "bus", and to "go by bus", and something else related to the bus.

\section{Research results}

The efficiency of use of emoji in the educational process is witnessed by the experiment's results. It was conducted among: the students of the first course of Donetsk National University of Economics and trade named after Mykhailo Tugan-Baranovsky in Kryvyi Rih city (60 persons), the pupils of Kryvyi Rih 
secondary school No.10 (50 persons). Such choice was made to indicate the age, status and informational features for emoji use. The results of approbation in the University showed $70 \%$ of desire to work with the texts containing emoji while the pupils from schools on the contrary showed 95\% of desire. Such difference should be explained with a difference in educational programs of the university and school, professional orientation, statusness of pupils of educational institutions of a certain age. The students perceived more positively the practical tasks with the use of emoji: testing, quizzes, - as one of the alternative forms for check of learned material, study and rest in a game form. The use of emoji had a positive effect in the growth of success of the experiment's participants (in average on 20\%), faster and better material assimilation, time saving. During the inquiry of the audience regarding the convenience of use of this development on a 5 -point scale, where 1 is a lack of understanding and perception, and 5 is a complete understanding and perception, we obtained the following results: 1 - 0\%; 20\%; thirty\%; 4 - 32\%; 5 - 68\%. The survey findings witness about complete perception of the audience development.

\section{Conclusions and perspectives of further researches}

So, the use of emoji in the educational process is an evidence for efficiency as from methodical, as from the technical point of view. The informational progress brings in our life corrections, changes not only the thinking, but also the means of communication, language of communication. The growth of volume of the informational flows created emoji, which became as a symbol of communication of the XXI century. A rapid expansion of emoji in different spheres of common life showed not only an efficiency and usefulness of their use, but also a possibility for their application in education. The recoding of educational texts with the use of emoji significantly reduces the amount of educational material, decreases the volume of educational material, decreases a load on the course participant. The emoji language awakens interest, activates the activity of the least active listeners, activates logical and associative thinking, promotes the formation of creativity. The practical tasks (based on the game form) indicate not only the wide variation of the forms of knowledge control, but also an increase in organized nature of the students, their interest in work. On the technical side, the use of smartphones, tablets, computers, opens an access to the study of material in any time and in any place, it solves one of the problems of most modern Ukrainian schools - distraction of a student on a smartphone during a class.

The realization of described model in the technical aspect is possible with the use of modern messangers: Viber, WhatsApp, Facebook Messenger, Skype. They allow you to use emoji in work and at the same time be a platform for the 
study of material, work in chat etc. The use of the wide set of emoji is possible thanks to free information resources of companies: Google, Aple, Google Drive, Emoji Keyboard Plug, etc.

The directions for future research lies in the use of emoji in the other disciplines, the extension of forms and methods for lessons realization, development of distance study, analysis of psychological mechanisms of influence on the listener, an activation of logical and associative thinking.

\section{References}

All on emoji. Festive test of Ukrainians in emoticons and symbols. Retrieved from https://zaxid.net/ves_na_emodzi_n1401515

Dubrovskaya, O. (2016). Emoji as a means of non-verbal youth communication. Vestnik BSU. Series 4: Philology. Journalism. Pedagogics, 2, 101-103.

Google has released a game for searching emoji around itself. Retrieved from https://delo.ua/business/google-vypustila-igru-dlja-poiska-emoji-vokrug-sebja-342267

Kane, M. (2018). Emoji marketing art: 7 smart examples from top brands. Retrieved from https://blog.hubspot.com/marketing/emoji-marketing-examples

Kitova, E. (2016). Communication in the Internet environment and the "universal language" Emoji. Theoretical and Practical Issues of Journalism, 5(4), 654-664.

Kondratieva, T. (2018). More than a third of teachers use Emoji, memes and gifs during class. Retrieved from https://life.ru/t/\%D0\%B2\%D0\%B0\%D1\%83/1039697/uzhie_bolieie_trieti_piedaghogh ov_vo_vriemia_zaniatii_ispolzuiut_emodzi_miemy_i_ghifki

Marissa, K. (2016). Lesson Idea: Developing Emoji Critical Reading Skills Retrieved from http://www.edutainme.ru/post/emoji-reading

Nosyrev, V. (2018). Code table. International Aviation Emergency Gesture Alarm. Retrieved from https://sites.google.com/site/skolazizniru/home/religioznaa-bezopasnost/izucenieobz-v-skole/turizm-v-skole/uzly/kodovaa-tablica-mezdunarodnaa-aviacionnaaavarijnaa-zestovaa-signalizacia

Osadcha, K., \& Chemerys, H. (2018). Formation of computer science bachelor's graphic competency while studying the prototification of soft interfaces. Information Technologies and Learning Tools, 5(67), 104-117.

Romanukha, O. (2018). Graphic interface as a didactic component of the electronic textbook. Information Technologies and Learning Tools, 5(67), 1-10.

Spotlight in iOS supports emoji search. Retrieved from https://www.macdigger.ru/ news/post/spotlight-v-ios-podderzhivaet-poisk-po-emodzi

Ukhanaieva, A. (2018). Emoji: More Than Emoticons. Retrieved from https://apptractor.ru/ marketing-monetization/emoji.html

Yandex. Translator» learned Emoji. Retrieved from https://mel.fm/novosti/4350689-yandex 